\title{
Expériences de carcéralité
}

Sortie de prison, grande pauvreté, hébergement d'urgence (France)

\author{
Gilles Chantraine* \\ https://orcid.org/0000-0002-4760-8879 \\ Laura Delcourt* \\ https://orcid. org/0000-0001-7684-9471
}

\begin{abstract}
Il ne faut pas voir en [la prison] la hautaine forteresse qui se referme sur les grands seigneurs de la révolte ou sur une sous-humanité maudite, mais la maison passoire, la maison de passe, l'inévitable motel. MICHEL FOUCAULT, préface à Leurs prisons, de Bruce Jackson, 1975.
\end{abstract}

La sociologie de la prison contemporaine est marquée du sceau de la diversité. Diversité des terrains tout d'abord, puisque nous assistons à un décloisonnement des aires géographiques, et au développement de recherches stimulantes, par exemple, sur les prisons asiatiques et africaines (voir par exemple Morelle, 2019; Martin, 2015). Diversité des objets ensuite: l'époque est lointaine où la recherche se focalisait presque exclusivement sur la relation surveillant-détenu et la production négociée de l'ordre carcéral. Certes cette relation reste l'élément nucléique de la prison. Mais il s'agit tout autant aujourd'hui de s' intéresser à la transformation des architectures (Scheer, 2016; Milhaud, 2017), aux usages sociaux du droit en détention (Galembert et Rostaing, 2004; Bouagga, 2015), aux temporalités et à la diversité des expériences individuelles de réclusion, aux pratiques de soin somatiques et psychiatriques (Lancelevée, 2016), à l'évasion (Martin et Chantraine [dir.], 2018), à la culture, aux études, à l'expérience de la maternité en prison (Cardi, 2014), aux rapports sociaux de sexe (Rambourg, 2013), aux transformations du métier de surveillant (Bennet, Crewe et Wahidin, 2013), ou encore au traitement de la "radicalisation" (Chantraine [dir.], 2018) etc.

* Universidade de Lille, Lille, França. 
Parmi cette diversification des objets et des focales théoriques, l'approche de la prison en termes de "déspécification" de l'institution constitue, aujourd'hui, l'une des voies les plus heuristiques. Par l'usage de l'expression "déspécification", il ne s'agit pas de nier les singularités de l'institution. Sécuritaire, guerrière, mortifère et pathogène: les caractéristiques structurelles d'une prison sont souvent les mêmes, et constituent une spécificité irréductible à toute forme de comparaison et d'analogie avec d'autres institutions ou d'autres mondes sociaux. Il n'en reste pas moins que la prison, bien souvent, "ne fait que transposer, voire exacerber au cœur de la détention, les inégalités sociales et les capacités d'initiative différentielles à l'œuvre à l'extérieur de la prison" (Chantraine, 2003, p. 382). Il s'agit alors d'objectiver la participation active de la prison "à des formes nouvelles de domination qui la dépassent tout en tendant vers elle, auxquelles la prison s'adapte aisément parce qu'elle les renforce” (Chantraine, 2004, p. 22). Par exemple, la récente thèse de Charles Kakule Kinombe (2019) sur les prisons congolaises est édifiante à cet égard. Outillé par les analyses convaincantes d'Olivier de Sardan (2001), il démontre comment l'activité des surveillants est caractérisée par le "privilégisme" (soit le fait de profiter des avantages de sa fonction, tel un usage abusif du téléphone), la "privatisation interne" (soit le fait de bénéficier systématiquement d'appointements supplémentaires dans l'accomplissement de sa tâche) et le "clientélisme" (politique, clanique), soit des processus et des pratiques qui structurent la culture bureaucratique privatisée de la société congolaise en général.

Il s'agit également de quitter la question de la "clôture" (clôture des théories pénales, clôture de l'institution), pour travailler plutôt sur les processus socio-pénaux de construction sociale de la population carcérale, et sur les trajectoires de celles et ceux qui l'expérimentent en tant que détenu.e.s. Les analyses en termes de gestion différentielle des illégalismes" (Foucault, 1975), ou de "filières pénales" (Aubusson de Cavarlay, 1985), permettent, entre autres choses, de comprendre l'imbrication de processus inégalitaires dans le fonctionnement du système pénal depuis le contenu et la formulation même des lois jusqu'aux tris sélectifs opérés aux différentes étapes du processus pénal.

Une dimension spécifique de l'approche en termes de "déspécification" concerne l'association socio-symbolique entre prison et quartier, association à l'intérieur de laquelle se dessinent des expériences de vie marquées du sceau de la "carcéralité", et ce par-delà les murs de la prison (Chantraine, 2004). Dans une variante archétypale, aux États-Unis, prison et hyper-ghetto s'assemblent dans une "symbiose fatale" pour devenir l'institution reproductrice des rapports sociaux de race (Wacquant, 2001). Comme le résume Manuela Ivonne Cunha: 
[...] the old congruence between the physical limits of the institution and social and symbolic boundaries can no longer be taken for granted. To understand inmates' perceptions, interactions and experiences, it becomes necessary to shift the focus from the "prison in context" to the interface between inside and outside, so as to capture the webs of relations and meanings which flow across and remake both worlds. Only by setting the neighborhood and the prison in analytical continuity can we take into account the emerging translocality of carceral social life (Cunha, 2008, p. 346).

Nous voudrions ici prolonger le travail de déspécification de la prison, non plus en portant attention aux liens entre quartiers populaires et prison, mais en s' intéressant aux conditions de sortie de prison des détenus les plus pauvres et à leurs efforts pour trouver un logement à l'issue de leur détention. Cette contribution s'appuie sur les résultats d'une étude qualitative financée et menée en partenariat avec la Fondation Abbé Pierre $^{1}$ (Chantraine et Delcourt, 2019), qui désirait avoir un aperçu de la situation quant à l'accès et au maintien du logement des personnes placées sous main de justice. Au cours de cette recherche, qui a duré une année et qui s'est achevée en 2017, nous avons mené 44 entretiens semi-directifs auprès de professionnels dans trois régions françaises: des Conseillers Pénitentiaires d'Insertion et de Probation (CPIP), des travailleurs sociaux de centres d'hébergement, et plus généralement des professionnels de l'insertion (chargés de mission, directeurs de structures...).

$\mathrm{Si}$, on l'a dit, les études sociologiques sur l'univers carcéral se sont considérablement diversifiées, il est tout à fait frappant de constater le nombre très faible de recherches portant sur la sortie de prison. Cette absence est pour le moins paradoxale, puisque si la prison est censée "réinsérer" les personnes qui y sont recluses, il paraît dès lors incontournable de s' intéresser à "l'après” prison. Absence plus remarquable encore, il n'existait, avant la recherche initiée par la Fondation Abbé Pierre, aucun document, analyse ou ouvrage portant de manière globale sur le rôle du logement et de l'accès au logement dans les processus d'insertion ou de réinsertion. La majorité des travaux recensés porte sur le profil des détenus et des sortants de prison, sur l'accompagnement social qui leur est proposé (pendant leur détention et à leur sortie), sur les difficultés de leur prise en charge et les obstacles à leur réinsertion, notamment professionnelle. Les problématiques propres à la question du logement sont pourtant nombreuses: quelle est la situation des personnes au regard du logement à leur sortie de prison (rue, habitat précaire, hébergement, logement etc.)? Qu'est-ce qui

1. La Fondation Abbé-Pierre pour le Logement des Défavorisés (fap), reconnue d'utilité publique depuis 1992, a pour mission de permettre à toute personne démunie d'accéder à un logement décent et à une vie digne, quels que soient le montant de ses ressources et sa situation sociale. Les auteurs tiennent à remercier les interlocuteurs de la fap avec qui nous avons échangé tout au long de cette étude. 
empêche une personne d'accéder à un logement à sa sortie? De façon plus générale, quels sont les freins à l'œuvre pour se maintenir ou accéder à un logement (individuels, collectifs, institutionnels)? Quels impacts cela peut-il avoir sur le conjoint ou la famille (impayés si la personne détenue était contributeur des ressources du couple, de la famille, résiliation du bail possible pour fait de délinquance d'un de ses membres, déménagement pour se rapprocher du lieu de détention ou pour échapper aux stigmates etc.)? Comment les proches/la famille arrivent à se mobiliser pour garder le logement d'un de ses membres incarcérés?

Dans le cadre de cette contribution, nous ne chercherons pas à répondre à toutes ces questions et renvoyons au rapport de recherche pour cela (Chantraine et Delcourt, 2019). Il ne s'agira pas non plus d'offrir une vue représentative de l'ensemble des expériences et des pratiques décrites dans les entretiens. Il s'agira ici, plus modestement, de pointer trois dimensions structurantes de l'expérience de celui qui sort de prison, et qui devraient nous inciter à poursuivre l'effort de déspécification analytique de la prison. Nous montrerons, tout d'abord, comment, pour les détenus les plus désaffiliés, le passage par la prison s'apparente moins à un "choc carcéral", selon une expression utilisée autant par les professionnels que par les sociologues, qu'à un épisode banal d'une existence précaire. Comme le résume Philippe Robert, "une trajectoire déjà très désaffiliée peut rester insensible au coup de pied de l'âne pénal: impossible de tomber plus bas" (Robert, 2005, p. 102). La prison peut même devenir un refuge protecteur de conditions de vie à l'extérieur pires qu'en prison. Ensuite, nous expliquerons comment la sortie de prison est marquée d'une impréparation et d'une incertitude extrême, incertitude qui prolonge la peine et l'emprise sur les corps des justiciables; contre le réductionnisme interprétatif qui consisterait à voir dans la libération la fin du châtiment légal, il nous faut au contraire observer comment la sortie de prison, telle qu'elle s'effectue concrètement, fait partie intégrante du dispositif punitif. Enfin, nous décrirons une réalité souvent sous-estimée dans les études s' intéressant aux modalités de sorties de prison des détenus, selon laquelle le détenu trouve un hébergement à sa sortie de prison. Celui-ci peut, en effet, être soumis à une pluralité d'exigences, à des micro-punitions et plus largement à un milieu de vie qui prolonge son expérience de carcéralité.

\section{Banalité de l'enfermement et prisonniérisation}

Si l'entrée en prison est régulièrement décrite et expérimentée comme un "choc", ce portrait risque en retour d'invisibiliser d'autres réalités, selon lesquelles une partie des personnes qui traversent la prison, au cours de leur trajectoire sociale, pourraient, paradoxalement, trouver dans l'institution pénitentiaire une béquille permettant de 
supporter ou d'échapper à une existence particulièrement difficile à l'extérieur. Ces expériences subjectives sont structurées et façonnées par des mécanismes macrosociologiques de sélection préférentielle, à travers l'appareil pénal, d'une population en situation de précarité économique et sociale, identifiée comme ayant des problèmes de santé mentale, peu diplômée, mal logée voire en recherche d'un logement ou d'un hébergement.

\section{Prison: la peine des pauvres}

En effet, d'après un échantillon de détenus de 1999, compris pour la première fois dans le recensement national français, on observe que la moitié a moins de 30 ans, que les trois quarts ont quitté lécole avant 18 ans, que le risque d'incarcération est multiplié par 16 pour ceux qui arrêtent l'école avant 16 ans comparés à ceux qui arrêtent avant 20 ans, que la moitié sont ouvriers, qu'on rencontre parmi eux deux fois plus de chômeurs que dans la population générale, que les cadres et les professions intellectuelles supérieures y sont très peu représentées, et enfin, que beaucoup sont nés à l'étranger. Parmi les sortants de prison, " $60 \%$ sont en situation de précarité socio-économique durable à l'entrée en détention” (De Beaurepaire, 2012, p. 126). Anne-Marie Marchetti (1997), auteure d'une des rares enquêtes sociologiques en France sur les situations de pauvreté en prison, observe que les détenus ayant davantage de moyens économiques ont de meilleures conditions d'existence en prison: ils effectuent les tâches les moins "ingrates" et les mieux rémunérées au sein de l'établissement. Or, 54\% des détenus vivent en dessous du seuil de grande pauvreté carcérale (soit 50 euros par mois, c'est-à-dire moins de $40 \%$ du revenu moyen en prison).

Pour Anne-Marie Marchetti (1997), si l'administration pénitentiaire répond aux besoins "primaires" comme l'alimentation, les détenus manquent de bien d'autres éléments nécessaires pour "vivre dignement et se positionner socialement de façon à ne pas être dominés", tels que des vêtements dignes, et plus globalement une indépendance financière. Ceux qui ne possèdent rien se voient alors attribuer des tâches par les autres prisonniers: ménage de la cellule, cuisine, circulation de produits... Par ailleurs, la vie en prison coûte cher: obligation, la plupart du temps, d'acheter soimême des vêtements, présence de plus en plus fréquente de produits que l'on peut se procurer si l'on en a les moyens (denrées alimentaires, produits d'hygiène etc.), location d'un téléviseur, d'un réfrigérateur...

Précarité sociale et précarité mentale sont également souvent associées, la présence de troubles mentaux étant largement supérieure en détention que dans la population générale. Cette double difficulté est régulièrement associée, dans les discours des professionnels, à l'expérience du sans-abrisme d'une partie de la population carcérale, 
la façonnant et renforçant l'engrenage de la précarité. Une assistante sociale, intervenant dans un service psychiatrique, en prison, résume l'association entre précarité sociale et précarité mentale d'une part, rue et prison d'autre part:

- Alors, dans la majorité des cas, c'est des personnes qui ont des parcours de vie dramatiques, souvent précaires: souvent il n'y a pas de logement. Pour le public que je suis, en particulier, il y a la double problématique de l'incarcération et de la maladie mentale [...]. C'est des gens qui n'ont des fois jamais travaillé, qui ne connaissent pas ce que c'est que le travail. C'est des gens qui, s'ils ont accédé à un travail, ça n’a jamais tenu dans la majorité des cas. C'est des gens qui n'ont pas de logement. S'ils en ont eu un, après, avec la maladie souvent, il n'y en a plus. C'est souvent des gens qui peuvent avoir été en centre d'hébergement ou dans des structures d'hébergement, plus qu’en appartement autonome. Dans la majorité des cas quand même, des sans-abri, beaucoup voilà.

\section{Quand la prison devient asile}

Sans surprise, le passage en détention est décrit par les professionnels comme une épreuve venant aggraver une situation déjà très précaire, épreuve dont résulte une déstabilisation profonde, tout autant sociale qu' intime. À l'instar d'Erving Goffman et de son concept d'institution totale (Goffman, 1968), les prisons ont été analysées sous l'angle du poids qu'elles exercent sur les reclus (tout comme d'ailleurs sur les professionnels qui y travaillent). Il s'agit ici du processus de prisonization (Clemmer, 1940), décrit par les sociologues de la prison, qui renvoie à l'analyse princeps du processus d'asilisation décrit par Goffman (1968). Robert Castel a bien résuméle phénomène:

L'asilisation exemplifie ce processus spécial d'adaptation à un univers claustral où le compromis de l'homme et de l'institution dans un temps immobile réalise la symbiose passive de l'initiative et de la répétition: la réussite des expédients journaliers et les conquêtes de détail qui améliorent le statut précaire du malade supposent l'intériorisation de la contrainte, si bien que la meilleure adaptation à ce milieu telle qu'on l'observe chez les vieux hospitalisés équivaut à l'impuissance à vivre dans tout autre milieu (Castel, 1989, pp. 31-43).

Cette prisonization progressive est d'autant plus saillante que la sortie s'avère brutale, notamment lorsque la violence du foyer d'accueil surpasse la violence carcérale elle-même:

- Je l'ai revu, je lui ai donné des infos sur les points d'accueil [pour sans-abri] où il peut prendre une douche, où il peut aller chercher une carte pour se restaurer le midi etc. Les foyers 
où les [sans-abri] atterrissent généralement, ça ne lui allait pas, c’était trop violent. En fait, il déchantait parce qu'il se rendait compte: quand il est sorti, il a été confronté à la réalité et du coup il s'est retrouvé mal. Il m’a appelé en me disant: "ben Madame x, je veux revenir en prison" (Travailleuse sociale dans un service psychiatrique en prison).

Selon un processus déjà analysé dans une autre enquête sur les trajectoires des prisonniers (Chantraine, 2004; Fernandez, 2010), la détention devient protectrice d'un monde extérieur invivable. Plusieurs travailleurs sociaux témoignent ainsi de retours volontaires en prison (notamment l'hiver), ou, comme cet éducateur spécialisé dans un dispositif de logement réservé à des sortants de prison le résume, du processus selon lequel l'institution totale produirait un cadre contenant qui se suffirait à lui-même:

- Je mesure mes propos, et ça m’arrache la tête de dire ça ! Mais il y en a qui m’ont dit qu'ils étaient bien en détention. Leur addiction est coupée, c'est compliqué de trouver de l'alcool, ils sont contenus. Ils ont un hébergement, ils sont nourris. Et le gars, s'il a envie de jouer à la Playstation toute la journée, il joue à la Playstation toute la journée. Pour certains, ça leur va. C’est un constat. Et c'est compliqué quand on fait du social. Le gars, au bout d'un moment, quand il est revenu une fois dans la même maison d'arrêt, tout le monde le connaît: il tutoie les surveillants, tout est là (Travailleur social d’une association spécialisée dans l'insertion post-carcérale).

Nous le verrons plus loin, un paradoxe ici réside dans le fait que la prisonniérisation n'est pas principalement décrite comme le fruit d'une adaptation à la discipline carcérale. Certes, les descriptions des douleurs de l'enfermement continuent de façonner les récits de vie et les entretiens semi-directifs, chez les détenus comme chez les professionnels. Mais c'est davantage l'adaptation à l'oisivetéforcée qui constitue le moteur de la sur-adaptation à la prison davantage qu'une hyper-minutie des faits et gestes. Quoi qu'il en soit, si l'expression de “choc carcéral” (dont l'un des symptômes saillants est l'augmentation du nombre de suicide en début de détention) reste pertinente pour décrire l'expérience de nombreux détenus, elle est en décalage avec l'expérience des détenus les plus précaires qui oscillaient déjà, avant leur incarcération, entre différents lieux et institutions de gestion de la grande pauvreté: foyer, hôpital, rue etc.

Sortir: galère et incertitude

Le second paradoxe que nous voudrions mettre en exergue ici peut s'énoncer simplement: ce qui apparaît comme une "libération" (soit la sortie de prison) cons- 
titue une prolongation, d'avantage qu'une rupture, de la peine sociale induite par l'enfermement. Ce paradoxe se laisse décrire selon quatre processus complémentaires: (i) d'abord, les détenus pauvres rencontrent la même difficulté que l'ensemble des groupes sociaux les plus paupérisés: l'absence de ressources économiques rend difficile l'accès à un logement; (ii) cette réalité première s'articule à un problème supplémentaire pour les sortants de prison, dont le temps de détention n'a pas permis d'activer ou de maintenir l'accès à différents droits; (iii) par ailleurs, les plus pauvres des plus pauvres tendent à être écartés à la fois des aménagements de peine et des centres d'hébergements susceptibles d'accueillir les sortants de prison; enfin, (iv), l'incertitude pénale et, notamment, l'incertitude qui caractérise la date effective de sortie de prison (Chantraine, 2004) pèse et contrarie encore un peu plus les possibilités d'accès à un hébergement.

\section{(Absence de) logement et pauvreté}

Cécile Brousse analyse les mécanismes qui tendent à inscrire certains individus dans des trajectoires de "sans-domicile". "La faiblesse du revenu agit de deux manières. Soit elle est directement à l'origine de la perte du logement (expulsion, impossibilité de s'acquitter d'un loyer etc.), soit, elle rend très difficile l'accès à un nouveau logement lorsqu'on a quitté le sien pour une raison ou pour une autre (séparation, décohabitation, émigration, sortie d'institution comme une prison ou un foyer)" (Brousse, 2006, p. 43). Le manque de ressources économiques est la principale raison poussant les futurs sans-domicile à arrêter leur recherche de logement: difficultés à se déplacer, à avoir une tenue correspondant aux normes d'un entretien d'admission, à disposer de la caution d'une personne physique. Ceux qui n'ont pas les ressources suffisantes mais qui tentent toutefois des recherches de logement, essuient des refus.

D'autres, enfin, se voient proposer un logement mais doivent le refuser pour des raisons économiques: leur budget est trop limité pour les loyers proposés ou l'éloignement du logement entraînerait des frais de déplacements qu'ils ne pourraient assumer. De plus, les personnes dotées de revenus très faibles sont aussi en état d'infériorité sur le "marché matrimonial" ce qui réduit probablement leur possibilité de rencontrer une personne logée (ou non) et donc d'accéder plus rapidement à un nouveau logement (éventuellement par l'intermédiaire du secteur associatif), ce phénomène étant sans doute plus accentué chez les hommes (Idem, ibidem). 


\section{La suspension des droits}

Dans ce cadre général, le passage en prison aggrave les choses, ne serait-ce que parce que le fait d'avoir eu du mal à payer son loyer avant l'incarcération renforcera la difficulté à en trouver un nouveau à la sortie: les bailleurs tiennent à ce que leurs locataires soient ponctuels quant au paiement du loyer et préféreront des dossiers montrant des ressources fiables et la garantie du paiement. Plus généralement, les travailleurs sociaux ainsi que les Conseillers Pénitentiaires d'Insertion et de Probation (CPIP) décrivent souvent la difficulté que rencontrent les détenus à activer leurs "droits": revenu minimum, assurance maladie, aides au paiement des loyers etc. Dans ce cadre, l'accès à ces droits en prison constitue à la fois une gageure et le nerf de la guerre de l'insertion, a fortiori dans une situation où les personnes détenues sont souvent démunies des ressources pour les faire valoir: méconnaissance des démarches, illettrisme qui entrave la compréhension des documents, délais d'attente trop longs dans une situation d'absence totale de ressources économiques etc.

Ces démarches, lorsqu'elles ne sont pas encadrées par un tiers, peuvent se révéler très complexes pour certains sortants de détention. Un résident d'un centre d'hébergement, placé sous bracelet électronique, explique ainsi que les lenteurs et les exigences du système d'accès aux droits épuisent un certain nombre d'ex-prisonniers:

- Ce que je ne trouve pas normal, par contre, quand on sort de prison ou d'une longue peine, c'est que la prison ne commence pas à entamer les démarches au niveau administratif. C'est-à-dire, Sécurité Sociale, la CAF, heu Pôle Emploi, s'il y a eu une démarche de faite. Pour la Sécurité Sociale ça se débloque seulement ! Parce qu'ils voulaient la levée d'écrou... Mais la levée d'écrou je ne peux pas l'avoir avant le 20. Donc tout ça, il aurait fallu que le centre pénitencier fasse les premiers pas pour amorcer la chose mais...

- Avant que vous puissiez sortir?

- Voilà. Ils étaient au courant que...Parce qu'ils étaient au courant depuis janvier que je sortais, que je suis sorti le 25 mars. Donc moi je trouve qu'ils auraient dû commencer à lancer la démarche de leur côté. Et ça, y en a beaucoup qui récidivent parce qu'ils n’ont personne pour les accueillir derrière et ils pètent les plombs: parce que les papiers pour la [sécurité sociale] ils ont au moins trois dossiers comme ça sur moi. Ça ne suffisait jamais! Et il y a des mecs qui pètent les plombs à cause de ça.

\section{L'éviction des plus pauvres des aménagements de peine}

Par ailleurs, l'accès à un aménagement de peine est soumis à un certain nombre d'obligations auxquelles doivent correspondre les détenus: emploi ou formation 
pour tout le temps de l'aménagement de peine, hébergement en concordance avec les exigences des magistrats. Or, celles-ci sont difficiles à obtenir en prison. De plus, les travailleurs sociaux attendent des prisonniers, implicitement ou explicitement qu'ils soient en "capacité d'autonomie", capacité qui s'exprime notamment par l'expression d'une motivation à demander de l'aide, dans le cadre d'un projet de sortie. Ainsi, au croisement d'une idéologie de "l'activation individuelle" et de la réalité selon laquelle chaque CPIP se sent débordé et en charge d'un trop grand nombre de dossiers, les individus les moins en "capacité", ou les "moins demandeurs", sont souvent exclus des dispositifs d'aménagements de peine et des aides pour accéder à un logement:

- Il y en a qui sont vus une ou deux fois au cours de leur peine, parce qu'on ne peut pas faire autrement. Et du coup, forcément, on passe à côté de plein de problématiques et on ne prépare pas du tout la sortie. Et, en plus, la plupart des personnes sont un peu perdues elles-mêmes, ne savent pas se prendre en main, sont pas demandeuses, se laissent un peu vivre... Et de la manière dont fonctionne l'administration pénitentiaire, bah ils passent complètement à côté quoi (CPIP, Centre Pénitentiaire).

Les détenus les plus pauvres sont ceux envers lesquels les actions seront les plus limitées, voire inexistantes. En détention, les droits comme notamment le revenu minium ou l'allocation-chômage sont suspendus. L'impossibilité, pour certains détenus ne travaillant pas en détention, de faire des économies en prévision de la sortie ( pouvant faciliter l'accès à un logement en payant une caution, l'avance de loyers, un hébergement temporaire à l'hôtel...) ou, pour reprendre les termes de Cécile Brousse, “[...] l'exclusion du droit de l'assistance sociale nourrit la pauvreté en détention et renforce la dimension de "parcours du combattant" de la libération" (Idem, p 13). Aussi, cette difficulté à se constituer un pécule de libération va tendre à exclure une partie des prisonniers de la possibilité d'aménagement de peine: les professionnels favorisent les dossiers offrant le plus de garanties de réinsertion.

La même CPIP explique la stratégie qu'elle met en place en sélectionnant méticuleusement les personnes qui bénéficieront d'un aménagement de peine:

- [...] enfin moi, sij’envisage un placement extérieur c'est quej’ai un petit peu ciblé la personne, c'est que je me dis: "bon il est vraiment en demande d'accompagnement, il est vraiment en demande qu'on l'aide". Et du coup c'est rare qu'ils mettent à mal le truc. [...] Parce qu'on les cible quand même. Moi je ne propose pas le placement extérieur [mesure d'aménagement de peine] à n'importe quel détenu. Même s'il est sans hébergement, même s'il est sans... $\mathrm{Si}$ je vois que le profil est trop bancal, que je ne lui fais pas confiance pour une permission de sortir... Parce que, là encore, il faut qu'il ait des permissions de sortir... Des structures 
demandent à voir la personne avant de donner une réponse favorable à l'intégration. Et qui dit permission de sortir, c'est soit on le laisse sortir tout seul: la structure vient le chercher mais il faut qu'on soit sûr qu'il va bien se comporter, qu'il ne va pas partir en cacahuète. Et nous, quand c'est nous, la plupart du temps qui assurons l'accompagnement... Enfin moi je ne propose pas à n'importe quel détenu où moi-même j'ai peur de prendre la voiture avec lui pour l'accompagner à la structure quoi. Donc normalement c'est un public ultra-ciblé, donc on ne prend pas trop de risques à les orienter vers ça.

La conservation des liens partenariaux entre les CPIP et les structures d'hébergement induit des stratégies de tris et d'éviction des “mauvais candidats". Leur confier des personnes jugées trop "instables" risquerait de les rendre réticents à accueillir des sortants de prison. L'accès aux aménagements de peine est limité du fait de la réticence des magistrats à les accorder en cas de non-conformité aux critères d'éligibilité, à cause du manque de structures d'hébergement, ainsi que de l'offre insuffisante de logements sociaux et d'emplois.

D’un autre côté, le spip (Service Pénitentiaire d'Insertion et de Probation) est soumis à la pression de conserver des liens avec les institutions d'hébergement. Ces dernières ont, en effet, le droit de refuser d'accueillir quelqu'un, pour maintenir l' "équilibre" des populations dans leur structure (ne pas accueillir uniquement des personnes sortant de détention). Nous l'avons évoqué précédemment, la sélection des détenus pouvant bénéficier d'une mesure d'aménagement de peine est, dans ce contexte, nécessaire au maintien des partenariats avec les espaces d'hébergement:

- Quelqu'un qu'on ne sent vraiment pas capable... Moi, quelqu'un que, je sais qu'il n'est pas capable de respecter une telle mesure, qui n'a pas de domicile, je ne vais pas créer une place en structure pour lui. Parce qu'après c'est du travail partenarial aussi. Ce sont des conventions. Déjà, en plus, s'il y a d'énormes retards de paiement, le Ministère de la Justice pour ces, pour ces places-là... On fait le tour des structures dans le genre, j'en ai visité un paquet, la plupart râlent contre nous, la Direction Interrégionale de Lille, parce qu'il y a des retards de paiement dans les chambres qui sont énormes. La plupart des structures veulent arrêter la convention avec le Ministère de la Justice à cause de ça. Si en plus on leur envoie des détenus, enfin des personnes placées qui sont, qui ont vraiment une problématique de comportement et qui vont mettre à mal la structure en deux jours, après, c'est sûr qu'on ne pourra plus jamais placer personne parce qu'ils diront: "bah non je refuse [...] Ils ont fait des mauvaises orientations". Donc c'est là aussi, c'est un monde du travail où on marche un peu sur des œufs à ne pas foutre en l'air un partenariat qui marche bien pour une personne où on a des gros doutes. Moi quelqu'un que je ne sens vraiment pas, bah c'est tout hein (CPIP, Centre Pénitentiaire). 


\section{Temps pénal, incertitude et peine sociale}

En 2004, lorsque l'un d'entre nous menait une recherche sur les expériences individuelles d'enfermement en maison d'arrêt, un détenu résumait d'une formule lapidaire l'incertitude radicale à laquelle était condamné le détenu: "la prison, on sait quand on entre, on ne sait pas quand on sort" (Chantraine, 2004). La peur qu'une autre affaire "tombe", l'incertitude quant aux remises de peine et aux aménagements de peine rend le temps de détention extrêmement incertain. Cette prolongation par l'incertitude du pouvoir pénal sur les corps et les individus entre en contradiction avec les exigences d'un travail d'insertion, qui consiste notamment à réserver une place dans un centre d'hébergement, et ce dans un contexte de pénurie.

- Le plus dur aussi, une des choses les plus dures avec ce job, c'est qu'on est... Les dates de sortie on ne sait jamais heu... Le gars on sait quand il rentre mais en gros on ne sait jamais quand il sort. Il peut sortir là, comme là 28 jours en avance, comme il peut rester des fois deux, trois ans sur place. Parce qu'une fois qu'ils sont sur place, il y a tout qui tombe et les mecs ils restent sur place. Et des fois c'est assez compliqué de bâtir des projets notamment d'aménagement de peine et de logement quand tout, au dernier moment, s'écroule parce qu'il y a encore une affaire qui est là (Un éducateur spécialisé travaillant dans une association spécialisée dans l'insertion post-carcérale).

L'incertitude de la date de sortie entraîne des difficultés de gestion du budget pour certaines associations investies dans un dispositif d'hébergement. Elles se portent, en effet, garantes auprès des bailleurs pour que certains logements soient mis à disposition du public précaire. En cas d'absence de paiement du loyer par l'usager, c'est l'association qui prend à sa charge la somme due. Une sortie de détention en milieu du mois, par exemple, inclut le règlement du mois entier (car le logement est réservé auprès du bailleur en attendant la fin de l'incarcération du condamné). Or, les aides au paiement des loyers, en France, ne sont prises en compte qu'à partir du jour d'entrée dans le logement. Le futur locataire, disposant de peu de ressources, n'a pas les moyens de s'acquitter entièrement de la somme. L'association est alors forcée de puiser dans ses réserves pour régler le loyer au bailleur.

- C’est-à-dire que là, les spip font leur rapport. Bon, pour le gars... Monsieur x doit sortir et nous, on leur fournit une attestation selon laquelle il va être pris dans "Trajet" [nom du programme spécifique]. C'est-à-dire qu’obligatoirement il aura le logement, l'accompagnement etc. Même si aujourd'hui, là, comme je lui ai dit, je ne suis pas en mesure de lui dire l'adresse exacte. Je ne suis pas en mesure pour l'instant, parce que le bailleur social nous l'a réservé à 
partir du 1er juillet. Voilà, à partir de juillet, c'est une magouille interne entre l'association x et le bailleur: s'il sort le, allez le 22 juillet, hé bien, nous, on paye du 1er au 22, et le gars paye du 22 au 31. Parce que sinon, ma foi, il aura les aides au paiement du loyer et c'est trop cher pour mettre au revenu minimum: une chambre de foyer c'est 480 euros. Le mec aurait eu ça; il ne peut pas quoi. [...]

- Et donc que ce ne soit pas vous qui aidiez à avancer les frais en fait. C'est ça?

- Ben ouais parce que c'est toujours pareil: nous on a un petit fond pour pallier ce genre de choses. Le fait d'absence de droit etc. Mais euh il reste déjà une quinzaine de personnes qui sont entrées. Plus on dépense d'argent et moins on a notre truc pour les prochains quoi.

Ces difficultés conjuguées rendent donc particulièrement difficile l'accès à un logement ou à un hébergement à la sortie de détention: manque de ressources économiques, contraintes administratives presque insurmontables, incompatibilité structurelle entre les institutions chargées de l'aide et la temporalité pénale. La sortie de prison prolonge la peine sociale associée à l'enfermement. Dans ce cadre, si de nombreux détenus ne mettent pas en place des dossiers de demandes d'aménagements de peine, ce qu'ils ne font pas toujours par manque d'information ou par manque de ressources sociales, c'est aussi pour prolonger le temps de détention et retarder la sortie. Par ailleurs, et c'est le troisième paradoxe que nous allons aborder, l'expérience de carcéralité ne s'achève pas avec la sortie de prison, et se prolonge dans d'autres institutions.

\section{D'une prison à l'autre: violence et discipline dans les hébergements}

L'expérience de carcéralité post-prison peut s’appréhender sous deux ensembles de phénomènes, qui constitueront l'ossature de cette dernière section. Dans un premier temps, il nous faut insister sur le fait que les sortants de prison, lorsqu'ils accèdent à un hébergement, accèdent parfois à un lieu qui concentre d'autres sortants de prison, lieu qui par ailleurs est parfois caractérisé par une vétusté et une saleté qui n'est pas sans rappeler les conditions de détention de certaines maisons d'arrêt. Ensuite, alors même que le passage en maison d'arrêt est souvent synonyme d'oisiveté forcée (il s'agit moins ici de surveiller et punir que de séparer et cloîtrer), l'accès et le maintien dans un hébergement à la sortie sont conditionnés à la soumission à de multiples disciplines et punitions éventuelles qui ne sont pas sans rappeler les anciennes disciplines carcérales. 
L'accès à un hébergement vétuste, précaire et insécurisant

Différentes structures d'hébergement sont à disposition, en France, lorsqu'une personne se retrouve sans logement, ni solution d'accueil au sein de la famille ou des proches: Centres d'Hébergement et de Réinsertion Sociale (CHRS), Centres d'Hébergement d'Urgence (CHU), Centre de Stabilisation, Logements et Chambres Conventionnés ALt (Aide au Logement Temporaire), Résidences Hôtelières à Vocation Sociale (RHVs), Centres d'Accueil pour Demandes d'Asile (Cada), Centres Maternels, Résidences Sociales, Maisons Relais, Foyers de Travailleurs Migrants, pour n'en citer que quelques-unes. L'ensemble de ces dispositions ont une vocation temporaire et sont censées mener les personnes à un logement dit "autonome".

Si les conditions de vie des différentes structures d' hébergement sont diversifiées, elles sont parfois déplorables et risquent de mettre en danger la sécurité de ceux qui y résident. Anne, une accompagnatrice emploi d'une association, décrit les conditions de vie très précaires des centres d'hébergement d'urgence. Si la structure qu'elle représente est spécialisée dans l'aide à l'insertion professionnelle, elle se retrouve à aider des personnes placées sous main de justice, ces dernières étant soumises à une injonction à occuper un emploi.

- Je vous dis, moi, j’ai une amie qui était en foyer. Le problème du dispositif c'est qu'ils se retrouvaient en milieu un peu fermé avec des gens qui avaient les mêmes problématiques. Donc je sais qu'il y a des trafics de drogue [...]. Alors c'est sûr, ils ont un endroit où dormir. Mais ça ne les sort pas de cette espèce de boue dans laquelle ils baignent depuis des années quoi, voilà. Après ça dépend aussi mais on a eu des sans domicile aussi.

- Qui étaient sans-abri avant d'être incarcérés?

- Oui, donc ben les sans-abri, le problème, c'est que même si on leur trouve un logement, s'ils ont été sans-abri pendant des années, ils ne tiennent pas dans le logement. Donc il faut qu'il y ait un accompagnement qui soit un peu long pour les habituer, les accoutumer: ce n'est pas simple quoi. Et après les foyers d'hébergement d'urgence, alors là, n’en parlons pas ! C'est... À Marseille c'est une horreur donc...

- Qu'est-ce que vous voulez dire? C'est surpeuplé, insalubre?

- Il y en a où il y avait même des viols. Il y en a qui étaient plein de puces; il y en a avec des punaises de lit, des choses comme ça. Moi, j’en ai un, il est arrivé, il était bouffé de la tête aux pieds. Donc je l'ai amené à la pharmacie, je lui ai acheté la pommade, pour qu'il se mette de la pommade quoi, voilà. Souvent ils préfèrent rester dans la rue...

Alors que nous posions la question, "qui était sans-abri avant d'être incarcéré?", l'accompagnatrice élargit immédiatement le "groupe-cible", à savoir non pas les 
personnes sortantes de prison sans domicile, mais "les sans-abri" en général; ici, les sortants de détention font d'abord et avant tout partie d'un groupe plus large. Et les problèmes qu' ils rencontrent seraient finalement similaires. Cette insalubrité et cette insécurité, décrites par cette professionnelle, ne sont pas sans rappeler les observations de Patrick Declerck, dans Les naufragés: avec les clochards de Paris (2001), où il décrit les conditions d'accueil de certaines structures d'hébergement d'urgence. Certains établissements sont insuffisamment équipés compte tenu des individus qui les fréquentent: matelas et lits non étanches sur des structures superposées, pour des occupants dont la consommation d'alcool entraîne souvent une incontinence, l'urine se retrouvant alors sur le lit du bas, désinfection insuffisante des draps alors que les sans domiciles sont parfois infestés de parasites, peur latente d'être volé ou agressé sexuellement, pour ne citer que quelques-unes de ses observations.

La concentration d'un certain type de public engendre parfois des difficultés de gestion des personnes accueillies, voire des événements violents, qui illustrent bien l'expérience de carcéralité:

- Enfin, le public "hommes isolés", on va prendre en CHRS "sortants d'incarcération". Ce qu’on va dire, c'est un classique: donc les CHRS généralistes accueillent énormément de personnes sortant d'incarcération. Et du coup, là, pour le coup, il y a des difficultés liées avec ce public-là. - De la cohabitation avec d'autres publics?

- Ouais. Surtout dans la cohabitation entre eux. Parce que, du coup, on reproduit le milieu carcéral dans les CHRS collectifs. Avec les mêmes personnes, puisqu' ils se connaissent. Ils étaient incarcérés au même endroit, au même moment. Donc ça reproduit la vie de l'incarcération au sein des collectifs. Donc c'est hyper compliqué. Alors là, il faudrait plus s'adresser à eux. On a deux gros centres d'hébergement CHRS généralistes hommes isolés; ils vous en parleront très bien. Régulièrement, ils nous alertent en nous disant: "là stop, c'est plus possible; on en a tant: ils s'appellent par leur numéro de matricule”. Ce n'est pas possible quoi (Une travailleuse sociale d'un service d'accès à l'hébergement).

Cette reproduction du fonctionnement carcéral, dans certains centres d'hébergement, est décrite par bon nombre de personnes interrogées au cours de l'étude: persistance de trafics, désignation d'un bouc émissaire, rapports de pouvoir et de domination.

\section{Discipline et punitions}

Le risque de surestimer la rupture entre rationalité pénale (la prison et sa logique punitive) et logique d'insertion (la réhabilitation après la prison) se joue également 
dans le contraste, ou justement le manque de contraste, entre le fonctionnement disciplinaire de la prison et l'assistance prodiguée dans les centres d'hébergement (Bérard et Chantraine, 2011). Certes les conditions de vie des centres d'hébergement ne sont pas restées inchangées. L'extension des horaires d'accueil dans les centres d'hébergement, par exemple, est un résultat concret de la mobilisation de militants, tels les "Enfants de Don Quichotte" (Brodiez-Dolino, 2009). Mais la question de la proximité entre

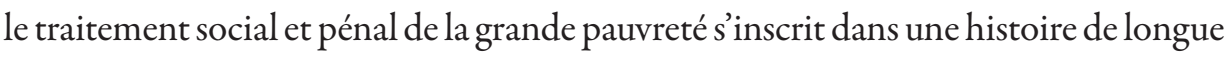
durée. La question récurrente dans les médias l'hiver, du refus des personnes à la rue de se rendre dans ces centres d'hébergement, indique qu'entre l'hostilité carcérale et la bienveillance de la solidarité, les lignes d'opposition sont sans doute variables et nuancées (Bérard et Chantraine, 2011). Comme l'exprime Patrick Bruneteaux (2007), "les méthodes carcérales ont été remisées, mais la précarité de l'accueil demeure, dans la logique d'un champ qui se situe en amont du champ de l'insertion. Ce sont des lieux où les droits existent peu, tandis que les mesures réglementaires s'exercent le plus souvent sous la forme d'interdits et de pouvoirs d'exclusion à l'encontre de toute personne jugée indésirable" (Bruneteaux, 2007).

Par ailleurs, l'accompagnement des résidents est décrit comme un élément primordial dans la prise en charge des sortants de détention dans les hébergements. Certaines structures offrent un accompagnement adapté aux besoins des personnes reçues. Une éducatrice travaillant dans une association mettant à disposition des places d'hébergement pour les sans-abri (surtout des hommes isolés ayant un passé carcéral), décrit l'aide qu'elle met en place à destination des anciens détenus résidant dans une structure d'hébergement (beaucoup de personnes accueillies ont un passé carcéral):

- On a la chance d'être une association à échelle très humaine hein, puisque que moi j’ai 27 personnes en tout. Donc on a un accompagnement, dans les premiers temps, intensif. C'est des personnes qu'on va voir tous les jours pratiquement et même plusieurs fois par jour. "Qu'est-ce que tu as fait ce matin? De quoi tu as besoin?” Enfin vraiment c'est limite du "nursing" au départ. "Est-ce que tu as pensé à la douche? Est-ce que tu as faim?" C'est vraiment heu, on reprend à zéro pratiquement l'éducation et le suivi quotidien. Donc là on est vraiment dans la confiance, dans le réapprentissage des bases de vie, de vie en société et l'accompagnement dans presque tout au départ. C'est: je dois l'accompagner physiquement à tous les rendez-vous; heu je dois expliquer ce qu'il se passe pour lui... C'est un travail qui est intensif. Là, on est dans le projet individualisé mais plus même! On est vraiment limite dans l'assistanat sur ces cas-là, lourds là. On a cette possibilité-là et heu petit à petit on les arme entre guillemets, j'ai envie de dire, en leur donnant des outils. Donc au départ, c'est nous qui rappelons, qui levons... Après bah hop on vérifie que... C’est le réveil qui a sonné, 
il s'est levé hein? Comme à la maison, j’ai envie de dire avec les enfants, au départ. Parce que tout est à refaire quoi. Et on arrive quand même à des gens qui redeviennent plus ou moins autonomes. Avec toujours ces carences affectives, ce suivi à l'extérieur mais qui reprennent un rythme de vie sociale.

Cet accompagnement passe aussi par une présence quasi permanente du personnel. Anne, l'accompagnatrice emploi de l'association, citée précédemment, introduit l'idée que certains sortants de détention ont besoin d'un "accompagnement renforcé" pour réapprendre à vivre dans un "intérieur":

- Oui, donc ben les sans-abri, le problème, c'est que même si on leur trouve un logement, s'ils ont été sans-abri pendant des années, ils ne tiennent pas dans le logement. Donc il faut qu'il y ait un accompagnement qui soit un peu long pour les habituer, les accoutumer: ce n'est pas simple quoi.

Les initiatives des acteurs de l'insertion, si elles ont pour objectif d'améliorer les conditions d'existence des personnes placées sous main de justice, se retrouvent parfois dans une position difficile. D'un côté, les professionnels ont pleinement conscience des effets pathogènes et désocialisant du passage par la prison, rendant le retour "à une vie normale dans un logement ordinaire” assez compliqué. Mais de l'autre, une certaine forme d'injonction consistant, précisément, à adopter tout un ensemble de "bonnes normes comportementales" et à respecter une discipline parfois très stricte en hébergement devient, précisément, une condition pour rester en hébergement. Les individus doivent d'abord acquérir les "compétences" jugées nécessaires pour être orientées vers un logement "autonome": bonne santé, autonomie, compétences diététiques et d'hygiène, entre-autres, ce qui peut parfois s'avérer contre-productif.

Il ne s'agit plus ici d'ériger le logement en droit fondamental, mais de le considérer sous une double dimension instrumentale: d'une part, le logement peut permettre de normaliser le comportement de la personne sortante de prison; d'autre part, la normalisation peut devenir une condition de l'accès ou du maintien dans un hébergement temporaire ou un logement durable. L'association entre le droit au logement et la conformité à une discipline institutionnelle stricte, qui n'est pas sans rappeler les normes de vie en prison, peut alors être assumée par les professionnels:

- Le CHRS est utile pour ça, pour leur réapprendre la vie. Une fois qu'ils ont eu 20 années d'incarcération, sans permission de sortir parfois, on a des gens qui sont en décalage. Décalage de leur vie, dont les diplômes ne valent plus rien. Ils ne savent plus exercer le métier 
qu'ils faisaient, qui ne sont plus en capacité physique de le faire etc. Et puis la détention c'est un mode de vie particulier avec des rites, beaucoup de... D'abord quand ils arrivent ici, très souvent ils nous appellent "chef". Tu es obligé de les reprendre là-dessus; ils disent: "il faut que je retourne dans ma cellule"... Donc on leur réapprend le terme de "chambre", de "domicile", des choses comme ça. Voilà, la prison les a façonnés comme ça. On leur demande d’avoir une tenue correcte. On n'est pas à la maison et on les resocialise petit à petit comme ça.

- Donc ils réapprennent les normes aussi de la société?

- Exactement, exactement. Donc s'ils ne sont pas inscrits au repas, ils ne mangeront pas, parce qu'il faut s'inscrire aux repas. S'ils ne sont pas à l'heure, ils ne mangeront pas. Ils n'étaient pas à l'heure... Enfin, toute une série de petites règles qu'ils ne comprennent pas et disent parfois qu’on est plus difficiles qu'en prison. Mais voilà, c'est le moyen pour eux de comprendre que l'accès à leur logement dépendra de tout ça quoi, de ces petites règles qu' il va falloir réapprendre petit à petit (Le directeur-adjoint d'un CHRS).

\section{Conclusion}

Lorsque Michel Foucault, dans l'exergue de cet article, parle "d'inévitable motel” pour caractériser la prison, et prétend que les détenus ne représentent selon lui ni "les grands seigneurs de la révolte", ni une "sous-humanité maudite", cela ne peut nous empêcher de penser à un problème central qui nous a interpellés tout au long de l'étude. Ce problème central consiste en ce que l'ensemble des acteurs interviewés ne semblent pas spécialement considérer les sortant-e-s de prison ayant des problèmes de logement comme un groupe distinct d'une population plus globale, en situation de grande pauvreté et mal-logée en quête d'un hébergement ou d'un logement. Une personne sans domicile qui serait passée par la prison ne présenterait ni plus ni moins de difficultés sociales qu'un autre sans-domicile: désocialisation, désaffiliation, grande pauvreté, fragilités psychiques. Le passage par la prison ne serait qu'une étape "banale" d'une trajectoire de grande désafiliation, qui, comme d'autres étapes tout aussi banales, ne viendrait finalement que reproduire ou amplifier des difficultés qui préexistent à l'incarcération. À trop insister sur l'effet de "rupture" porté par la rationalité pénale ou la singularité d'un système guerrier défensif, l’analyste risque en effet de scotomiser une réalité pourtant parfois essentielle de l'épreuve carcérale telle que la traversent bon nombre de détenus, particulièrement en maison d'arrêt, à savoir qu'elle ne constitue qu'une étape parmi d'autres d'une trajectoire de désaffiliation, d'exclusion et de pauvreté. 


\section{Références}

Aubusson de Cavarlay, Bruno. (1985), "Hommes, peines et infractions: la légalité de l'inégalité". L'Année Sociologique, 35: 275-309.

Bennet, Jamie; Crewe, Ben \& Wahidin, Azrini (eds.). (2013), Understanding prison staff. London, Wilan.

Bérard, Jean \& Chantraine, Gilles. (2011), Sortir, s'en sortir: l'innovation pénale dans les aménagements depeine. Rapport de recherche, Emmaüs/Haut Commissariat aux Solidarités Actives.

Bouagga, Yasmine. (2015), Humaniser la peine: enquête en maison d'arrêt. Rennes, PUR.

Brodiez-Dolino, Axelle. (2009), Emmaüs et l'Abbé Pierre. Paris, Presses de Sciences Po.

Brousse, Cécile. (2006), "Devenir sans-domicile, le rester: rupture des liens sociaux ou difficultés d'accès au logement”. Économie et Statistiques, 391-392: 35-64.

Bruneteaux, Patrick. (2007), "Les politiques de l'urgence à l'épreuve d'une ethnobiographie d'un SDF". Revue Française de Science Politique, 1 (57): 47-67.

Cardi, Coline. (2014), "Les quartiers mère/enfant: l'“autre côté" du dedans? Protection et surveillances des mères et prison de femmes". Penal Field, 11.

Castel, Robert. (1989), “Institutions totales et configurations ponctuelles". In: JospeH, Isaac (org.). Le parler frais d'Erving Goffman. Paris, Minuit, Arguments, pp. 31-43.

Chantraine, Gilles \& Delcourt, Laura. (2019), Favoriser l'accès et le maintien dans le logement des Personnes Placées Sous Main de Justice. Paris, Les Cahiers du Logement, FAP.

Chantraine, Gilles; Scheer, David \& Depuiset Marie-Aude. (2018), "Enquête sociologique sur les 'quartiers d'évaluation de la radicalisation' dans les prisons françaises”. Rapport DAP-CNRS, Clersé, Université de Lille.

Chantraine, Gilles \& Kaminski, Dan. (2008), "Rights in prison: institutional police, juridical activism, democratic struggles". Champ Pénal/Penal Field, Séminaire Innovations Pénales. Disponível em http://journals.openedition.org/champpenal/7033.

Chantraine, Gilles et al. (2012), "Space and surveillance in a prison for minors”. Politix, 97 (25): 125-148.

Chantraine, Gilles. (2003), "Prison, désaffiliation, stigmates: l'engrenage carcéral de l'inutile au monde' contemporain”. Déviance et Société, 4 (27): 363-387.

Chantraine, Gilles. (2004), Par-delà les murs: expériences et trajectoires en maison d'arrêt. Paris, Presses Universitaires de France.

Chantraine, Gilles. (2005), "Prison and sociological perspective: for an off-center critical analysis". Penal Field, 1.

Chantraine, Gilles. (2010), "French prisons of yesteryear and today: two conflicting modernities. A socio-historical view". Punishment \& Society, 1 (12): 27-46.

Clemmer, Donald. (1940), The prison community. Boston, Christopher Publishing. 
Cunha, Manuela. (2008), "Closed circuits kinship, neighborhood and incarceration in urban Portugal”. Ethnography, 3 (9): 325-350.

De Beaurepaire, Christiane. (2012), "La vulnérabilité sociale et psychique des détenus et des sortants de prison”. Revue du Mauss, 40 (2): 125-146.

Declerck, Patrick. (2001), Les naufragés, avec les clochards de Paris. Paris, Plon.

Fernandez, Fabrice. (2010), Emprises. Drogues, errance, prison: figures d'une expérience totale. Bruxelles, Larcier.

Galembert, Claire \& Rostaing, Corine. (2004), “La prison comme 'laboratoire’ des usages sociaux du droit". Droit et Société, 87.

Goffman, Erving. (1968), Asiles. Paris, Minuit.

JACKSON, Bruce. (1975), Leurs prisons. Paris, Plon.

Kakule Kinombe, Charles. (2019), La surveillance partagée et capitalisée: ethnographie d'une prison en RD Congo. Louvain-la-Neuve, thèse (doctorat), Faculté de Droit et de Criminologie de l'uc de Louvain.

LANCELEveE, Camille. (2016), "Quand la prison prend soin? Gérer les troubles mentaux dans un établissement pénitentiaire allemand pour femmes”. Sociétés Contemporaines, 103 (3): 91-110.

Marchetti, Anne-Marie. (1997), Pauvretés en prison. Toulouse, Erès.

Martin, Tomas \& Chantraine, Gilles. (2018), Prison breaks: toward a sociology of prison escape. London, Palgrave MacMillan.

Martin, Tomas. (2015), "Witnessing and accessing Ugandan prisons: fieldwork within a post-colonial bureaucracy”. In: DrAKe, Deborah et al. (orgs.). Palgrave handbook of prison ethnography. Hampshire, Palgrave, pp. 424-441.

Milhaud, Olivier. (2017), Séparer et punir: les prisons françaises: mise à distance et punition par l'espace. Paris, CNRs.

Morelle, Marie. (2019), Yaoundé carcérale: géographie d'une ville et de sa prison. Lyon, ENS.

Olivier De Sardan, Jean-Pierre. (2001), "La sage-femme et le douanier: cultures professionnelles locales et culture bureaucratique privatisée en Afrique de l'Ouest'. Autrepart, 4 (20): 61-73.

Rambourg, Cécile. (2013), Origines et évolution de la féminisation de l'administration pénitentiaire. Paris, Enap.

Robert, Philippe. (2005), La sociologie du crime. Paris, La Découverte.

Scheer, David. (2016), "Conceptions architecturales et pratiques spatiales en prison: de l'investissement à l'effritement, de la reproduction à la réappropriation”. Revue de Science Criminelle et de Droit Pénal Comparé, 2 (2): 419-427.

Wacquant, Loïc. (2001), "Symbiose fatale: quand ghetto et prison se ressemblent et s'assemblent". Actes de la Recherche en Sciences Sociales, 139: 31-52. 


\section{Résumé}

Expériences de carcéralité: sortie de prison, grande pauvreté, hébergement d'urgence (France)

Cet article se base sur une enquête qualitative sur les conditions de sortie des détenus les plus pauvres en France et de leur recherche d'un logement. 44 entretiens ont été menés avec des professionnels de l'insertion. Déplaçant le regard théorique depuis l'effet de clôture de l'enfermement vers l'analyse des continuités expérientielles de part et d'autre des murs de la prison, l'analyse replace l'épreuve de l'enfermement dans une expérience de "carcéralité" qui l'englobe et la déplace. Dans cet article, il s'agit moins de résumer l'ensemble des résultats concernant l'accès au logement des sortants de prison en France, que de se focaliser sur trois paradoxes qui restent souvent dans l'ombre de la sociologie de la prison, et qui appellent à en revisiter certaines routines. D'abord, l'incarcération elle-même constitue moins un "choc carcéral" qu'une expérience banale dans une trajectoire de grande précarité; par ailleurs, la "loi d'airain", qui veut que les conditions de détention soient pires que les conditions de vie du travailleur pauvre à l'extérieur, trouve ses limites dans l'expérience de celui pour qui la prison devient un asile protecteur. Ensuite, la sortie se caractérise par une impréparation et une incertitude radicale dont pâtissent tout particulièrement les détenus les plus pauvres. Il s'agit moins d'une “libération" qu'une plongée et un retour à la précarité la plus crue. Pour le dire en d'autres termes, la libération ne met pas fin à la peine, elle en fait partie intégrante. Enfin, l'accès à un hébergement, qui se présente souvent comme la seule option, prolonge l'expérience carcérale: concentration d'anciens détenus, insalubrité, violence et discipline forment la trame de la vie quotidienne en leur sein.

Mots-clés: Prison; Pauvreté; Carcéralité; Sortie de prison; Déspécification; Hébergement.

\section{Abstract}

Prison experience: release from prison, extreme poverty, emergency housing (France)

This paper is based on a qualitative survey on the conditions of release of the poorest prisoners in France and their search for housing. 44 interviews were conducted with social worker and other professionals. Shifting the theoretical view from the "closing effect" of confinement to the analysis of experiential continuities on either side of prison walls, the analysis replaces the confinement experience in an experience of "carcerality" that encompasses and displaces it. In this article, it is less a question of summarizing all the results concerning access to housing for people leaving prison in France than of focusing on three paradoxes that often remain in the shadow of the sociology of prison, and which call for a review of certain routines. First, incarceration itself is less a "prison shock" than a banal experience in a very precarious trajectory; moreover, the "less eligibility" which requires that prison conditions be worse than the living conditions of the poor worker outside, finds its limits in the experience of the person for whom prison becomes a protective asylum. Secondly, the release is characterised by a lack of preparation and radical uncertainty, particularly for the poorest prisoners. It is less a "liberation" than a return to the worst precariousness. In other words, release does not end the sentence, it is an 
integral part of it. Finally, access to accommodation, which is often the only option, extends the prison experience: concentration of former prisoners, insalubrity, violence and discipline form the backbone of daily life.

Keywords: Prison; Poverty; Carcerality; Prison release; Despectification; Housing.

\section{Resumo}

Experiências de carceralidade: saída da prisão, pobreza extrema, alojamento de urgência (França) Este artigo baseia-se em uma pesquisa qualitativa sobre as condições de soltura dos prisioneiros mais pobres da França e sua busca por moradia. Foram realizadas 44 entrevistas com profissionais de integração. Deslocando o olhar teórico sobre os efeitos do período de confinamento em direção à análise das continuidades experienciais dos dois lados dos muros prisionais, a análise recoloca a passagem pelo encarceramento no interior de uma experiência de carceralidade que a engloba e a desloca. Neste artigo, trata-se menos de resumir todos os resultados relativos ao acesso à moradia para pessoas que foram soltas das prisões na França, do que focar em três paradoxos que frequentemente permanecem nas sombras da sociologia da prisão, e que obrigam a revisitar certas rotinas. Primeiro, o próprio encarceramento é menos um "choque da prisão" do que uma experiência banal em uma trajetória altamente precária. Além disso, o "conceito arraigado", segundo o qual as condições de detenção são piores que as condições de vida do trabalhador pobre no exterior, encontra limites na experiência da pessoa para quem a prisão se torna um asilo protetor. Em segundo lugar, a saída é caracterizada por um despreparo e uma incerteza radical, que afeta particularmente os presos mais pobres. É menos uma "libertação" do que um mergulho e um retorno à precariedade mais crua. Em outras palavras, a liberação não coloca fim à pena, é parte integrante dela. Por fim, o único acesso disponível a um alojamento prolonga a experiência carcerária: concentração de ex-prisioneiros, insalubridade, violência e disciplina formam a trama da vida cotidiana em seu seio.

Palavras-chave: Prisão; Pobreza; Carceralidade; Saída da prisão; Desespecificação; Alojamento.

Texto recebido em 22/8/2019 e aprovado em 19/9/2019.

DOI: $10.11606 / 0103-2070 . t s .2019 .161341$

Gilles Chantraine é pesquisador no Clerse, Centre Lillois d'Études et de Recherches Sociologiques et Économiques (F-59000), na Université de Lille (CNRS, UMR, 8019). E-mail: gilles. chantraine@univ-lille.fr.

LAURA DELCOURT é pesquisadora no Ceries, Centre de Recherche Individus Épreuves Sociétés (F-59000), na Université de Lille. E-mail: laura.delcourt@univ-lille.fr. 